\title{
La Ciencia política en Colombia: DE VOCACIÓN A DISCIPLINA*
}

\author{
Ana María Bejarano \\ Universidad de TORONTO, CANADÁ \\ María Emma WilLs \\ Universidad Nacional de Colombia, Colombia
}

A Dora Rothlisberger, In Memoriam

\begin{abstract}
Resumen
En las últimas dos décadas, la ciencia política en Colombia no sólo se abrió paso, sino que ganó autonomía y prestigio en el campo académico. Este acumulado ha requerido un deslindamiento de las pasiones partidistas y de las polarizaciones intelectuales, así como la utilización de estrategias para sobrevivir el embate de la guerra. Hoy existen más de una docena de departamentos de ciencia política en el país, con programas de pregrado, especializaciones y maestrías. Ha crecido el número de revistas que divulgan los resultados de investigación, cuya agenda se ha expandido. Sin embargo, para garantizar la calidad de la enseñanza y la investigación, se requiere propiciar un mayor debate entre las/os politólogas/os colombianas/os, fortalecer los vínculos con la comunidad académica internacional e institucionalizar los mecanismos de evaluación y regulación.
\end{abstract}

\begin{abstract}
During the last two decades, political science in Colombia has not only cleared its way, but has also gained in autonomy and academic reputation. This has entailed a greater distance from party passions and intellectual polarizations, as well as the use of strategies to resist the wave of violence in the country. There are over a dozen political science departments, with a diversity of undergraduate, specialization and master's programs. The number of journals has also grown and the research agenda has expanded. However, in order to guarantee the quality of teaching and research there needs to be a greater debate among Colombian political scientists, the links with the international academic community must be strengthened, and the mechanisms of evaluation and regulation institutionalized.
\end{abstract}

\section{PALABRAS CLAVE • Ciencia Política • Colombia • Historia de la Disciplina • Investigación}

El estudio de la política, en tanto ciencia social independiente ${ }^{1}$, comenzó en Colombia en 1968 con la creación del Departamento de Ciencia Política en la Universidad de los Andes. Durante estas tres décadas y media, la enseñanza y la investigación en ciencia política han dejado de ser monopolio de un departamento en particular. Tal diversificación ha venido acompañada de un

* Agradecemos la colaboración de Celenis Rodríguez en la recolección de la información utilizada en la elaboración del presente artículo. Agradecemos también los comentarios de dos lectores anónimos.

1 Resulta preciso distinguir, como lo hace Torres Velasco $(1994,124)$, "los estudios políticos" -en donde convergen contribuciones de varias disciplinas-, de "la ciencia política" en tanto campo relativamente autónomo del conocimiento. Nuestro artículo se centra primordialmente alrededor de la evolución de la ciencia política en la segunda acepción, aunque haremos breves referencias a los cruces y fertilizaciones mutuas con otros aportes a la comprensión de lo político. Desafortunadamente, tal restricción nos obliga a dejar de lado algunas instituciones claves en el estudio de la política colombiana como el Centro de Investigación y Educación Popular, CINEP, fundado en 1973 por la Compañía de Jesús. 
proceso de consolidación de la politología como disciplina autónoma. Este proceso de crecimiento y diversificación sin duda viene acompañado de riesgos. La demanda de ingreso a la carrera ha aumentado notablemente desde principios de los años 90 , sin que se hayan consolidado los mecanismos formales e informales ${ }^{2}$ que garanticen su calidad; pese a la proliferación de departamentos y programas, a nivel nacional no se han fijado ni aplicado de manera rigurosa estándares mínimos de exigencia. Lo cual implica que existe aún una mayor urgencia para generar espacios de debate público donde los resultados de la docencia y la investigación en este campo sean discutidos a fondo entre colegas y estudiantes. La creación de instancias de autorregulación académica es, por tanto, uno de los retos centrales: es allí de donde deben surgir los filtros que legítimamente contribuyan a regular y mantener la calidad de la profesión.

En las siguientes páginas se describe de manera somera la evolución de la ciencia política en Colombia y se sugieren algunas explicaciones al respecto. En particular, el que esta disciplina haya encontrado su propio lugar en el campo académico, corre paralelo a un profundo pero aún inacabado proceso de secularización y desideologización de la mirada sobre la acción y las instituciones políticas en Colombia. Estas transformaciones se pueden medir no sólo en términos de la autonomía que adquiere la ciencia política frente a instituciones como la Iglesia Católica, sino también por la distancia que los estudios políticos realizados desde la universidad han adquirido frente a los partidos, sean ellos los tradicionales, los de izquierda o los actores armados; y aun frente a los movimientos sociales y la sociedad civil. Así, la ciencia política, en estas décadas, ha pasado de defender causas políticas o paradigmas ideológicos, a comprender y explicar procesos para, a partir de ese conocimiento histórico, sugerir criterios o estrategias para la acción política.

\section{I. ÉPOCA DE PIONEROS (1968-1986): ABRIENDO EL PASO}

No es mera coincidencia que el primer departamento de ciencia política hubiese aparecido en la Universidad de Los Andes, una universidad joven (fundada apenas dos décadas antes), privada, secular, con una orientación claramente "modernizante"3. En medio de un contexto político conflictivo, caracterizado por el cerramiento del régimen operado por los acuerdos del Frente Nacional, el surgimiento de una crítica cada vez más radical al arreglo institucional post 1958 y una movilización estudiantil en plena efervescencia, la naturaleza privada de la Universidad le permitió al naciente departamento distanciarse de la militancia radical, por entonces tan extendida en los campus universitarios, especialmente en las facultades de sociología donde se practicaba la "so-

2 Por mecanismos formales se entienden todas aquellas iniciativas institucionales que, a través de una regulación nacional, establecen y aplican parámetros de calidad para los programas de educación superior. Por mecanismos informales se señalan todos aquellos incentivos y desestímulos que generan la propia academia y el mercado laboral frente a los egresados de los diversos programas.

3 Un documento bien interesante, que permite apreciar el contexto que ofrecía la Universidad de Los Andes en ese momento, es la propuesta preparada en 1964 por Fernando Cepeda para el CHEAR (Council for Higher Education in the American Republics), donde se presenta la iniciativa de crear un programa de Ciencia Política en la Universidad. Ver Cepeda (1983: 17-35).

$4 \quad$ Véase Hernández Lara, Jorge (1983: 97-101), citado en Leal Buitrago (1994: 103). La relación entre la sociología y la ciencia política en el país ha sido estrecha y compleja. La ciencia política colombiana le debe su auge, en parte, a la crisis de las facultades de sociología. La crisis de la sociología como disciplina, sin embargo, constituye una pérdida grave para el país y por otro lado le roba a la ciencia política la posibilidad de un intercambio fructífero con esta disciplina. Sobre la trayectoria de la sociología en Colombia ver Segura Escobar y Camacho Guizado (2000). 
ciología comprometida"4. Por otro lado, su naturaleza secular le permitió una aproximación más moderna, racional y por sobre todo no-confesional a los problemas y fines de la actividad política. Esto explica que el departamento haya logrado sobrevivir y consolidarse en una época donde las facultades de ciencias sociales, tanto públicas como privadas, fueron víctimas de la polarización y de la estigmatización que llevaron a muchas de ellas al cierre definitivo

Lo cual no quiere decir, sin embargo, que la Universidad de Los Andes haya sido totalmente inmune a la polarización política que caracterizó las décadas de los 60 y 70 . Con efecto retardado, a principios de los 70, el campus de Los Andes también se convirtió en un "campo de batalla político". En 1973, las directivas expulsaron a un puñado de líderes estudiantiles, terminaron el contrato de varios profesores y clausuraron la carrera de Bellas Artes; paradójicamente, optaron por conservar la de ciencia política ${ }^{5}$. Pese a que se mantuvieron al margen de las protestas estudiantiles, los profesores del departamento también protagonizaron polarizaciones intelectuales durante estas épocas. Aun cuando ningún docente usó su cátedra para hacer proselitismo político, el cuerpo profesoral, por momentos, sí profesó adhesiones incondicionales a los paradigmas intelectuales (marxismo versus estructuro-funcionalismo) que entonces se confrontaban como campos teóricos irreconciliables. Este clima sólo comenzaría a cambiar hacia mediados de los 80.

El Departamento de Ciencia Política de la Universidad de Los Andes fue el único programa docente de pregrado en el país por casi veinte años ${ }^{6}$. Allí se educó la primera generación de politólogos propiamente dichos; allí se originaron los primeros proyectos de investigación de la política colombiana, deslindados tanto de las exigencias del Estado, como de la influencia de los partidos tradicionales ${ }^{7}, 0$ de la Iglesia Católica. En la investigación como en la docencia, el departamento de la Universidad de los Andes se inclinó desde el comienzo por el modelo norteamericano: muchos de sus profesores iniciales fueron visitantes venidos de diversas instituciones norteamericanas ${ }^{8}$; algunos de los profesores colombianos habían recibido formación de postgrado en Estados Unidos. Gracias al hecho que en la universidad no existía una Facultad de Derecho en el momento de la fundación del departamento, su currículo tuvo desde el principio un perfil claramente politológico: deslindado tanto de los estudios jurídicos como de las humanidades, con énfasis en metodologías y temas típicamente asociados con el estudio de la política desde la perspectiva de las ciencias sociales ${ }^{9}$. Los primeros proyectos de investigación realizados por el departamento pueden calificarse, con unas pocas excepciones ${ }^{10}$, como mainstream, tanto por los temas abordados (comportamiento legislativo, partidos políticos y grupos de presión, elecciones y participación electo-

5 La Universidad estuvo cerrada desde octubre de 1972 hasta el primer semestre de 1973, debido a los disturbios ocasionados en los predios universitarios. Se sancionaron setenta y dos estudiantes, siete de los cuales fueron expulsados de la universidad. Ver Espinal (1998: 35 y 59-60)

6 En 1975 se abrió un programa de postgrado en la Facultad de Estudios Interdisciplinarios de la Universidad J averiana en Bogotá; una década después se extendió este programa de maestría a la sede de Cali. Ver Torres (1994: 127).

7 Con algunas excepciones, como Fernando Cepeda, y Mario Latorre, quienes mantuvieron siempre vínculos activos con el Partido Liberal.

8 Ver la lista de profesores visitantes del Departamento, en Sánchez (1994: 140-141).

$9 \quad$ Ver Autores Varios (1983: 102-103). "Primer Programa de Estudios en Ciencia Política". Tal deslinde frente al estudio del derecho no era posible en las universidades más tradicionales, tanto públicas como privadas, donde el estudio de lo político había estado siempre subordinado al estudio de lo jurídico.

10 Entre las que se cuentan el trabajo pionero de Paul Oquist sobre la violencia en Colombia (1980) y la obra de Francisco Leal Buitrago, enfocada en el Estado, con particular atención al tema militar, con un fuerte acento marxista. El mejor ejemplo de su trabajo de esa época es el libro Estado y Política en Colombia (1984). 
ral, por ejemplo), como por el enfoque utilizado, inspirado fundamentalmente en el funcionalismo y el conductismo en boga por entonces en los Estados Unidos ${ }^{11}$. La novedad, sin embargo, no radicaba en el tema, ni en la aproximación metodológica; lo verdaderamente novedoso era el acercamiento a los fenómenos políticos desde una perspectiva no partidista, más moderna y

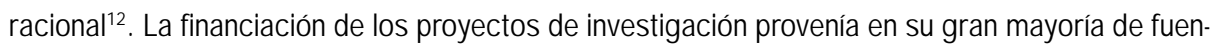
tes norteamericanas: la Fundación Rockefeller y la Fundación Ford, así como el Canadian Institute for Internacional Development (CIID) financiaron buena parte de las investigaciones originalmente realizadas por miembros del departamento ${ }^{13}$.

Pese a la dificultad para obtener el reconocimiento social de una profesión hasta entonces desconocida en el país, el Departamento de Ciencia Política de la Universidad de los Andes perseveró. En retrospectiva, los dos mayores logros alcanzados en esas décadas fueron haberle abierto camino a la disciplina, y haber consolidado un programa de licenciatura de excelente calidad ${ }^{14}$. No obstante, en aras de ofrecer un balance equilibrado, también habría que señalar las metas no alcanzadas hasta finales de los 80: pese a sus dos décadas de existencia, el departamento no logró consolidar, en términos de investigación, una producción académica sólida, coherente y sostenida ${ }^{15}$. La dificultad para afianzar la investigación en estos 20 años estuvo estrechamente ligada con otra ausencia: durante ese período, el departamento no contó con ningún medio de divulgación de sus investigaciones en la forma de una publicación periódica de calidad. Finalmente, pese a muchos esfuerzos, ensayos y errores, tampoco se logró, a lo largo de las primeras dos décadas, la consolidación de un programa de estudios de postgrado en la disciplina ${ }^{16}$.

\section{TIEMPOS DE CAMBIO (1982-1990): INVESTIGAR Y PUBLICAR, LOS NUEVOS IMPERA- TIVOS}

El predominio del Departamento de Ciencia Política de la Universidad de Los Andes en el campo de los estudios políticos comenzó a cambiar a mediados de los años 80. Por un lado el Centro de Investigación y Educación Popular (CINEP) le dio un renovado impulso a la investigación en el campo político y continuó con la labor de difusión de los resultados investigativos en su revista Controversia.

11 Sobre el tema de las investigaciones ver Leal, Rothlisberger y Sánchez (1983: 53-57).

12 Por la misma época, otras cuantas instituciones de carácter privado: la FEI de la Universidad J averiana, el Instituto SER de investigaciones y Fedesarrollo, le dieron un gran impulso a los estudios electorales en Colombia.

13 Las fuentes de financiación para investigaciones no son objeto de análisis detallado pero aparecen recurrentemente en los balances de la disciplina aparecidos en dos libros editados en conmemoración de la fundación del departamento: Departamento de Ciencia Política (1983) y Sánchez (1994).

14 Vale pena anotar aquí que pese a la reciente proliferación de departamentos de ciencia política en el país, tanto en la universidad pública como en la privada, el departamento de la Universidad de Los Andes es el único que ha recibido el reconocimiento del Consejo Nacional de Acreditación.

15 Las razones para esta deficiencia son múltiples y ameritan un estudio más detenido. Ellas van desde la ausencia de medios de divulgación (que anotamos más adelante), hasta la escasez de recursos para la investigación, pasando por el limitado número de académicos dedicados a tal actividad. También tiene que ver con el hecho de que las universidades no solían ofrecer a sus docentes los estímulos necesarios para adelantar investigaciones de alta calidad (descargas en docencia, bonificaciones, fondos de investigación).

16 La maestría en la Universidad de Los Andes se inició en 1975 y graduó una primera generación de estudiantes, entre los que se encuentran varios reputados investigadores. Dos promociones más adelante, sin embargo, la maestría entró en receso. Finalmente reinició labores en 1989 y ha mantenido su continuidad hasta ahora; en 1998 se realizó una gran reforma curricular. Ver Postgrados (s.f.). 
En 1982, por su parte, la Fundación Foro Nacional por Colombia inició labores tanto de investigación como de promoción, y en 1986 inició la publicación de la Revista Foro, dedicada a difundir ensayos e investigaciones de académicos tanto nacionales como extranjeros, sobre temas relativos a la democracia, los movimientos sociales, la descentralización y la participación ciudadana.

Fue en ese mismo año, pero esta vez en un campus universitario, cuando se fundó el Instituto de Estudios Políticos y Relaciones Internacionales (IEPRI) en la Universidad Nacional de Colombia. En su seno se reunieron sociólogos, historiadores, abogados y politólogos de trayectoria para "fortalecer la reflexión política en la universidad pública, apoyada en el conocimiento y el análisis científico de la materia"17. En sus orígenes, esta reflexión se concibió como un estímulo "para la lucha por una democracia real" y alimentó la revista Análisis Político, la cual se ha mantenido sin interrupciones hasta hoy. Además de esta producción periódica, los miembros del Instituto divulgaron sus hallazgos investigativos a través de libros y de su activa participación en foros, seminarios académicos y en programas de opinión en los medios. La audiencia de la producción del Instituto no ha sido ni prioritaria ni exclusivamente la comunidad estudiantil, sino el amplio terreno de formación de la opinión pública. Desde sus inicios, el Instituto no quiso circunscribirse a los temas exclusivos de la ciencia política, sino que definió su campo de investigación como el de los estudios políticos. De allí su orientación interdisciplinaria, tanto en lo temático como en la formación de los investigadores que lo componen. Finalmente, a diferencia de lo que aconteció en el departamento, la mayoría de los investigadores del Instituto realizaron sus estudios superiores en la academia europea, menos tajantemente disciplinar y más cercana a las humanidades que la norteamericana.

Desde el punto de vista temático, en sus primeros años el IEPRI se volcó hacia el estudio de la violencia, sobre todo en su dimensión política. Dejó su impronta en el debate público con libros como Colombia: Violencia y Democracia (Comisión de estudios sobre la violencia, 1987), un informe auspiciado por el Ministerio de Gobierno durante la presidencia de Virgilio Barco, en el que participaron activamente varios de sus miembros. Con esta publicación se formalizó un nuevo tipo de vínculo entre Estado e intelectuales: de la sospecha o el desconocimiento mutuo, la relación transitó hacia un momento de diálogo y cooperación. Por otra parte, las articulaciones entre Estado, régimen político y violencia, las relaciones desiguales entre regiones y centro, las estrategias de los actores armados, las políticas públicas ante el reto guerrillero, las concepciones jurídicas subyacentes a las contiendas armadas, la irrupción del narcotráfico, las continuidades y las rupturas entre las violencias de ayer y las de hoy fueron todos temas que pasaron por la lupa investigativa de los miembros del Instituto ${ }^{18}$.

A finales de la década de los 80 , el énfasis en la dimensión investigativa de la ciencia política recibió un nuevo impulso, esta vez desde otra entidad pública: la Universidad de Antioquia fundó el Instituto de Estudios Políticos (IEP) en 1988. Este instituto, como el IEPRI, se definió como interdisciplinario, pero en contraste con el primero combinó investigación con docencia casi desde sus inicios. En 1990, el Instituto Colombiano para el Fomento de la Educación Superior (ICFES) aprobó el programa de Maestría en Ciencia Política del IEP que se ha mantenido hasta el día de hoy ${ }^{19}$. 


\section{LOS ÚLTIMOS 15 AÑOS (1990-2005): CRECIMIENTO Y DIVERSIFICACIÓN}

Durante la década de los 90, la ciencia política en Colombia vivió un proceso dinámico de multiplicación y consolidación, el cual acarrea sus propios riesgos. Basta con mirar la explosión de programas dedicados al estudio de la disciplina para constatarlo (ver tabla 1): desde 1994 se han creado 14 nuevos programas de pregrado ${ }^{20}$ en universidades tanto públicas (9) como privadas (5). Un dato interesante tiene que ver con la distribución regional de estos nuevos departamentos: menos de la mitad de ellos (6) están ubicados en Bogotá. El resto (8) han surgido en la provincia - usualmente en los más importantes centros regionales: Medellín (3) y Cali (1). Pero también en unas cuantas ciudades intermedias tales como Manizales, Popayán y Santa Marta. Claramente, Bogotá ha perdido su monopolio como centro de formación en la disciplina- , lo cual no deja de ser una tendencia bien interesante. Sin negar la importancia de este proceso de descentralización y diversificación, tal multiplicación de programas conlleva un riesgo en la medida en que no viene encuadrada en una regulación rigurosa, ni existe la exigencia de una acreditación. Por ejemplo, de todos los programas de ciencia política registrados hoy en día, sólo uno está acreditado formalmente ${ }^{21}$.

A partir de 1994 se han registrado ante el Ministerio de Educación 17 nuevos programas de pregrado en Ciencia Política en el país. En el tabla 1 incluimos 14 de ellos (más el de la Universidad de los Andes). Los otros tres (localizados en la Corporación Universitaria Republicana, la Universidad de Boyacá y la Fundación Universitaria Los Libertadores) fueron excluidos del cuadro porque aparecen registrados como programas de derecho y ciencias políticas, lo cual contradice nuestra concepción de la ciencia política como una disciplina autónoma tanto del derecho como de las humanidades y otras ciencias sociales.

21 De 243 programas acreditados a nivel nacional, sólo uno es en ciencia política (el de la Universidad de Los Andes). Ver www.cna.gov.co 
TABLA 1: Colombia: pregrados registrados en ciencia política

\begin{tabular}{|c|c|c|c|c|}
\hline Universidad & Programa & Municipio & $\begin{array}{l}\text { Fecha } \\
\text { Registr }\end{array}$ & Duración \\
\hline Universidad de los Andes & Ciencia Política & Bogotá D. C. & 1970 & 9 Semestres \\
\hline U. Nacional de Colombia & Ciencia Política & Medellín (Antquia.) & 1994 & 8 Semestres \\
\hline U. Nacional de Colombia & Ciencia Política & Bogotá D.C. & 1995 & 8 Semestres \\
\hline Pontificia U. J averiana & Ciencia Política & Bogotá D.C. & 1995 & 10 Semestres \\
\hline U. Externado de Colombia & Gob. y RR. II. & Bogotá D. C. & 1995 & 10 Semestres \\
\hline Universidad del Cauca & Ciencia Política & Popayán (Cauca) & 1996 & 8 Semestres \\
\hline $\begin{array}{l}\text { Colegio Mayor de Nuestra } \\
\text { Señora del Rosario }\end{array}$ & $\begin{array}{l}\text { Ciencia Política } \\
\text { y Gobierno }\end{array}$ & Bogotá D. C. & 1996 & 10 Semestres \\
\hline Universidad del Valle & $\begin{array}{l}\text { Estudios pol. y } \\
\text { resoluc. conflictos }\end{array}$ & Cali (Valle) & 1998 & 5 años \\
\hline U. Militar Nueva Granada & RR. Il. y Estudios Pol. & Bogotá DC & 2000 & 10 semestres \\
\hline ESAP & Ciencias Pol. y Adm. & Bogotá D. C. & 2000 & 10 Semestres \\
\hline Pontificia U. J averiana & Ciencia Política & Cali (Valle) & 2001 & 10 Semestres \\
\hline Universidad del Magdalena & $\begin{array}{l}\text { RR. Il. y estudios } \\
\text { pol. }\end{array}$ & $\begin{array}{l}\text { Santa Marta } \\
\text { (Magdalena) }\end{array}$ & 2002 & 10 semestres \\
\hline Universidad de Antioquia & Ciencia Política & Medellín (Antquia.) & 2003 & 8 Semestres \\
\hline EAFIT & Ciencias Políticas & Medellín (Antquia.) & 2003 & 10 Semestres \\
\hline U. Autónoma de Manizales & Ciencia Política & Manizales (Caldas) & 2003 & 9 Semestres \\
\hline
\end{tabular}

Fuente: www.mineducación.gov.co/SNIES

Adicionalmente, han surgido media docena de programas de "especialización en ciencia política", los cuales típicamente duran dos o tres semestres. Estos se distribuyen equitativamente entre la universidad pública (3) y la privada (3), pero tienen como característica el estar situados mayoritariamente (todos excepto uno) fuera de Bogotá; con la excepción de dos programas ubicados en Medellín y Barranquilla, todos los demás se encuentran en ciudades intermedias tales como: Ibagué, Tunja, Arauca y Bucaramanga. De alguna manera comienza a vislumbrarse el impacto de la descentralización que se ha llevado a cabo en Colombia en las últimas dos décadas: a la par con la distribución del poder político se ha creado también una demanda por comprender y analizar los procesos políticos regionales y locales.

Finalmente, también se han multiplicado los programas de Maestría: aparte del programa de Los Andes que luego de una suspensión de más de diez años reinició labores en 1989, existen hoy en el país seis programas de maestría en estudios políticos, los cuales se distribuyen de manera equitativa también entre la universidad pública (3) y privada (3). Geográficamente, las maestrías se encuentran concentradas en las grandes ciudades: tres en Bogotá (en Los Andes, la J averiana y el IEPRI de la Universidad Nacional), dos en Medellín, una en Cali y otra en Manizales. 
Como consecuencia natural de esta proliferación de centros de enseñanza, el número de graduados en ciencia política se ha multiplicado exponencialmente en las últimas dos décadas ${ }^{22}$. Lo cual no quiere decir que todos opten por el estudio de la política como actividad profesional: estos siguen siendo una minoría. Lo que sí se advierte como consecuencia de la creciente competencia por los cargos de docencia e investigación disponibles es una tendencia a la mejor calificación de los profesores/investigadores: hoy en día tiende a ser la norma y no la excepción, que los profesores de planta de los diversos departamentos cuenten al menos con estudios de maestría, a la par que ha aumentado considerablemente el número de docentes/investigadores con título de doctorado ${ }^{23}$. En recientes convocatorias para la vinculación de docentes en departamentos de ciencia política en universidades tanto públicas como privadas, se ha vuelto común la exigencia del título de doctorado como requisito para ingresar a la carrera profesoral ${ }^{24}$.

Claramente, sin embargo, no son las universidades ni los centros de investigación ${ }^{25}$ los que han absorbido la mayor parte de esta masa de politólogos egresados de los numerosos programas aquí reseñados. El mercado de trabajo también ha sufrido transformaciones radicales en las dos últimas décadas: hace apenas 20 años, el estudiante recién egresado del departamento de Ciencia Política tenía tres opciones de trabajo: la docencia/ investigación en la universidad, el periodismo político, o la vinculación a la vida política activa -la cual, por supuesto, dependía de sus contactos con los políticos profesionales-. Hoy en día, las opciones se han multiplicado exponencialmente: des de la empresa privada, pasando por el mundo de las ONG y las organizaciones multilaterales, hasta una diversidad de cargos en el Estado, a nivel local, regional y nacional, los nuevos politólogos cuentan con una amplia gama de opciones laborales (Murillo y Ungar, 2000: 235). Hay que anotar, en particular, un cambio cualitativo en la relación entre el Estado y las ciencias sociales, relación que estuvo caracterizada por la mutua sospecha hasta principios de los años ochenta. Comenzando con la administración de Belisario Betancur (1982-1986), pero sobre todo durante los gobiernos de Virgilio Barco (1986-1990) y César Gaviria (1990-1994), las relaciones entre el Estado y la academia dieron un vuelco definitivo. No sólo se pasó del antagonismo al diálogo, sino que el Estado se ha convertido en el mayor empleador de los egresados de estos departamentos. Esto coincide con una expansión de la planta del poder ejecutivo (la crea-

22 El Departamento de Ciencia Política de la Universidad Nacional de Colombia, sede Bogotá, contaba en el segundo semestre de 2003 con 615 estudiantes. Ver www.unal.edu.co/derecho. En la sede de Medellín (Universidad Nacional), hay 320 estudiantes inscritos en la carrera. En el Departamento de Ciencia Política de la Universidad de Los Andes por su parte había 368 estudiantes inscritos en el 2004 a nivel de pregrado; en el primer semestre de 2005 se habían inscrito 42 estudiantes a nivel de licenciatura y 48 a nivel de la especialización. Ver http://c-politica.uniandes.edu.co/home.html. La Facultad de Gobierno, Ciencia Política y Relaciones Internacionales de la Universidad del Rosario cuenta con cuatro doctores (dos graduados en Alemania y Suiza, uno en España, y otro en Francia). El Departamento de Ciencia Política de la Universidad de Los Andes cuenta con nueve doctores (siete graduados de universidades norteamericanas, uno en Alemania, y uno en México). Ver http://zulia.colciencias.gov.co. En cuanto al IEPRI, de su planta de investigadores adscritos, en este momento cuenta con cinco doctores, cuatro de los cuales se graduaron en Europa y una en EEUU. Folleto IEPRI, s.f.

24 Ver el concurso docente (aún vigente) de la Universidad Nacional de Colombia, y la última convocatoria del Departamento de Ciencia Política de la Universidad de los Andes. La Universidad de Antioquia ha mostrado más flexibilidad en su contratación, y ha vinculado a personas con maestría pero con el compromiso de que emprendan estudios doctorales. Esta universidad, en un programa que ha probado ser altamente exitoso, apoya además a los recién ingresados a la carrera docente, con créditos y becas para que emprendan estudios de doctorado. Algo similar ocurre en la Universidad Externado de Colombia.

25 De 821 grupos de investigación acreditados por COLCIENCIAS en el área de humanidades, 56 están registrados como grupos que realizan investigación en el área de la ciencia política. De estos 56, cinco pertenecen a la categoría $A, 4$ a la B, 2 a la C, y los restantes 45 están 'reconocidos', lo cual indica que la mayoría aún se encuentra en proceso de formación. Ver http:// zulia.colciencias.gov.co:8081/grupos.war/ search/EnGrupolnvestigacion/ 
ción de múltiples consejerías presidenciales), la apertura de algunos organismos de planeación (como el Departamento Nacional de Planeación) a profesionales de disciplinas distintas a la economía, y finalmente con la descentralización: como se anotaba más arriba, no es sólo en el Estado central, sino también en las administraciones regionales y locales, donde los profesionales de las diversas disciplinas sociales (no sólo la ciencia política) han encontrado un nuevo nicho laboral26.

Ahora bien, volviendo a la producción de conocimiento científico en ciencia política, la tendencia es también hacia la diversificación de la agenda de investigación. A los temas tradicionales se han venido a sumar nuevas preocupaciones: movimientos sociales, sociedad civil, ciudadanía, formación de la esfera pública, descentralización, proceso constituyente, reforma del Estado y diseño institucional, resolución pacífica de conflictos y justicia transicional. Incluso los temas más tradicionales se abordan ahora desde una óptica distinta, nuevos marcos teóricos, distintas metodologías; el enfoque tradicional para el estudio de la historia política, por ejemplo, ha dado paso a investigaciones centradas en la formación del Estado y la nación (González et al., 2003); el estudio de las elecciones y los partidos políticos se hace ahora incorporando la teoría neo-institucional y herramientas como la teoría de juegos; la violencia se analiza en sus dimensiones micro, así como también se incorporan miradas comparativas. Por otra parte, las rígidas fronteras disciplinares se diluyen para dar un renovado impulso a investigaciones más interdisciplinarias, que combinan preocupaciones de la sociología, la antropología o la psicología política. Resta mucho camino por recorrer; pero sin duda, el paisaje de la producción académica en ciencia política es, hoy por hoy, mucho más rico y diverso que hace dos décadas.

Esta tendencia tiene su correlato en la expansión y diversificación de las publicaciones periódicas que cumplen la labor de divulgar la producción de los investigadores. Aunque el libro sigue predominando como forma principal de divulgación, la publicación en revistas especializadas ha ido ganando terreno en las últimas décadas. Tres revistas fundadas a mediados de los 80 han sobrevivido hasta hoy: se trata de Ciencia Política, Revista Foro y Análisis Político. A ellas se han sumado la Revista de Estudios Sociales y Colombia Internacional, ambas de la Universidad de Los Andes, Estudios Políticos de la Universidad de Antioquia, Reflexión Política de la Universidad Autónoma de Bucaramanga, Desafíos de la Universidad del Rosario, y la segunda etapa de Controversia del CINEP27.

Por otra parte, la tendencia a la diversificación también se ha extendido al campo de la financiación de la investigación: si en un principio la mayoría de los proyectos eran financiados por fundaciones norteamericanas, el panorama ha cambiado radicalmente en los últimos 20 años: la tendencia a la disminución de los fondos disponibles en las fuentes tradicionales ha obligado a diversificar la búsqueda de fuentes extranjeras, especialmente en Europa. Por otra parte, la participa-

26 Basta una mirada superficial a los equipos de gobierno de los actuales alcaldes de las principales ciudades del país (Bogotá, Medellín y Cali, por ejemplo) para confirmar la creciente presencia de politólogos, economistas, sociólogos, antropólogos y hasta filósofos en la nómina municipal. En cuanto al aparato central del Estado, los politólogos han venido a engrosar las filas de los abogados y los economistas en la formación de una incipiente meritocracia. Este hecho es desconocido por Serres (2005) en su reciente discusión sobre las élites en la administración pública colombiana.

27 De éstas, sólo Estudios Políticos (Universidad de Antioquia), Análisis Político (Universidad Nacional; Reflexión Política (Universidad Autónoma de Bucaramanga), la Revista de Estudios Sociales y Colombia Internacional (ambas de la Universidad de los Andes) aparecen indexadas en PUBLINDEX, el índice elaborado por COLCIENCIAS (ver a.colciencias.gov. c0:8080/lillium/htmIPublindex/ index.html" http:// huitaca.colciencias.gov.co:8080/lillium/htmlPublindex/ index.html.) Aun cuando varias de ellas tienen un gran prestigio en la comunidad académica, sólo una está clasificada en la categoría B y todas las demás están en la $\mathrm{C}$. 
ción de recursos nacionales en la financiación de la investigación ha aumentado considerablemente: desde comienzos de los años 80, el Instituto Colombiano para el Avance de la Ciencia, COLCIENCIAS, se ha convertido en una fuente de recursos y apoyo a las tareas de investigación, de innegable importancia. Más recientemente, este esfuerzo por parte del Estado ha sido complementado con recursos de la empresa privada, la cual ha invertido algunos recursos en la promoción de investigaciones bien directamente, o bien a través de fundaciones como la Fundación Corona, o la Fundación Ideas para la Paz, por ejemplo.

Desde el punto de vista de los institutos y centros de investigación, a partir de mediados de los 90 se han incorporado nuevos miembros a los equipos de investigadores. Estos nuevos investigadores le han dado impulso a nuevas temáticas -identidades sociales, formación de nación, memoria- 0 han tratado de aislar las miradas sobre ciertos fenómenos como el del análisis electoral y de los partidos políticos, del estudio de la violencia. Por otra parte, los análisis comparativos, ya sea con los países andinos, o con sociedades que transitan por guerras civiles o donde prosperan diversas economías ilegales, son objeto de un interés cada vez mayor.

Pese al fortalecimiento en términos de números, temática y composición, durante los años 90, y como consecuencia de la violencia en la universidad, se produjo una transformación del lugar desde el cual los investigadores y en general los académicos hablan de la situación colombiana y de la política en particular. Seis de los 19 docentes de planta del IEPRI se vieron obligados a salir del país, bajo la amenaza o la ejecución de ataques armados propiciados por distintos actores del conflicto. En Medellín, varios docentes de la Universidad de Antioquia fueron asesinados y por la misma época también cayó asesinado un profesor de la Facultad de Economía en el propio campus de la universidad. Frente a tal agresión, los académicos (en particular los miembros del IEPRI), optaron por seguir ejerciendo su labor pero bajo la consigna de ocupar un "bajo perfil" en sus actividades de extensión. Además, y probablemente sin ser muy conscientes de ello, los investigadores adoptaron posiciones mucho más desencantadas frente a la política y más cautas ante la guerra. Ante reiterados intentos frustrados de negociación y pacificación, la/os politóloga/os colombiana/os han dejado de buscar "la política correcta" o "el sujeto capaz", por sí solo, de conducir al país hacia la paz, y han tratado en su lugar de comprender la paradójica situación de una sociedad que se ahoga en la violencia, pese a una serie de esfuerzos reformistas llevados a cabo a partir de la década de los ochenta. Si en un principio, los estudiosos de la política trabajaban animados por la confianza en que el conocimiento científico podía sugerir las rutas más propicias para afianzar la democracia como el opuesto a la guerra, a finales de los 90 muchos de ellos han moderado sus expectativas y han aprendido dolorosamente que las reformas promovidas no siempre desembocan en los lugares y las situaciones deseadas. De las esperanzas a veces teñidas de ingenuidad y los análisis orientados por el deber ser de finales de los 60, la comunidad académica ha pasado a un cierto desencanto y a un afán por comprender el presente a partir de una mirada más realista de la propia historia.

\section{EL FUTURO: DESAFÍOS Y PERSPECTIVAS}

Pese al crecimiento registrado en las últimas dos décadas, el tamaño de la comunidad de politólogos sigue siendo pequeño ${ }^{28}$. El reto continúa siendo el de construir una masa crítica de estudiosos de

28 Los que sigue se inspira, parcialmente, en Bejarano (2000). 
la política, continuar los esfuerzos por ensanchar y calificar nuestra comunidad académica mediante el fomento de programas de pregrado y postgrado de alta calidad ${ }^{29}$, así como la consolidación de grupos y centros de investigación. En suma, ya logrado el establecimiento de la disciplina en el país, el esfuerzo debe dirigirse ahora a consolidar la profesionalización de la misma.

Este proceso debe ir de la mano de un esfuerzo consciente de conformar una verdadera "comunidad intelectual", proyecto que pasa sin lugar a dudas por el diálogo entre investigadores de la política. Pese a la diversificación y afianzamiento de las últimas décadas, los resultados de investigación no suscitan aún hoy el debate público y la discusión entre colegas que ameritarían ${ }^{30}$. La/ os politóloga/ os colombiana/ os, a diferencia de los historiadores, los sociólogos, los antropólogos o los filósofos, no tienen su propio congreso anual, y los centros de investigación siguen estando presos de tendencias endogámicas. El reto consiste en crear una cultura de debate crítico, oral y escrito, entre las pequeñas islas que conformamos el archipiélago de la ciencia política en este país. Las revistas que hemos mencionado en este artículo cumplen en buena medida con esta función de servir como vitrinas de exposición de las ideas, como centros receptores y transmisores de la crítica. Todavía falta, sin embargo, que nos leamos y nos critiquemos más. Y hace falta, sobre todo, un foro nacional donde se presenten, se discutan y se critiquen los resultados de investigación que produce la comunidad. En parte debido a la ausencia de tal escenario, las diferencias teóricas y metodológicas que dividen la disciplina y que en otros países son motivo de arduos y a veces álgidos debates, no se han hecho visibles ni explícitas en Colombia. Una razón adicional para explicar lo anterior, es el imperio de la coyuntura: en un país que parece desmembrarse a cada paso, resulta difícil - por no decir imposible- deslindarse del dramático desenvolvimiento de la situación política nacional para ahondar en debates sobre diferentes visiones de la disciplina.

Finalmente, el último reto consiste en garantizar el clima político e intelectual que permita el afianzamiento del pluralismo temático y metodológico. La nueva agenda de investigación es extensa: la globalización y su impacto en América Latina y en Colombia; el cambiante sistema interamericano y el nuevo rol de la OEA en la región; las perspectivas de un acuerdo de libre comercio de las Américas; las consecuencias regionales del conflicto colombiano; la explosión de identidades étnicas en la región andina y su impacto en el terreno político; los estudios de género y la construcción de ciudadanía, son algunos de los temas que se vislumbran en el horizonte. Además, debemos afrontar el desafío de aplicar nuevas miradas sobre viejos problemas tales como la violencia, la construcción de un orden democrático o el histórico predominio de los partidos tradicionales. Finalmente, se trata también de hacer más plural nuestra comprensión del mundo político, de diversificar el rango de actores presentes en el repertorio de la política: las mujeres, los indígenas, las poblaciones afro-colombianas.

29 En el momento no existe en Colombia ningún doctorado en ciencia política. Sin embargo, la Universidad Externado de Colombia está a punto de aprobar un doctorado en este campo, y en el IEPRI se está afinando un proyecto de doctorado en estudios políticos.

30 COLCIENCIAS ha empezado a generar estímulos para el intercambio investigativo entre centros de distintas regiones del país. En su última gran convocatoria - una financiación a cinco años por US \$1.7 millones de dólares para alianzas temporales- el objetivo era estimular la formación de comunidades académicas en torno a proyectos de investigación compartidos. Desafortunadamente, a pesar de que cuatro alianzas temporales clasificaron y fueron bien evaluadas en el área de Cultura, Instituciones y Desarrollo, el Consejo Nacional de Ciencia y Tecnología decidió otorgar los fondos a alianzas temporales de las áreas de la salud y la investigación en ciencias naturales. Ver: http:// www.colciencias.gov.co/ convocatorias/ convocatorias_cod.php?cod $=248$ 
En un país cruzado por múltiples violencias, es quizás un milagro que se haya logrado preservar, hasta hoy, una comunidad científica activa y diversa. Múltiples esfuerzos tanto individuales como institucionales han contribuido a generar este pequeño milagro. No cabe duda de la importancia de preservar este espacio de resistencia: al igual que otros espacios en la sociedad civil, la comunidad académica, con su capacidad para promover el debate y la crítica, es quizás el mejor antídoto contra la violencia.

\section{REFERENCIAS}

Autores Varios. 1983. La Ciencia Política y la Universidad de Los Andes. Bogotá: Departamento de Ciencia Política - Editorial Guadalupe Ltda.

Bejarano, Ana María. 2000. "Ciencias sociales en Colombia: Tres retos hacia el futuro". En Discurso y razón. Una historia de las ciencias sociales en Colombia, editado por Leal Buitrago y Rey. Bogota: Tercer Mundo Editores - Ediciones Uniandes - Facultad de Ciencias Sociales - Fundación Social.

Cepeda, Fernando. 1983. "La Ciencia Política como nueva disciplina". En La Ciencia Política y la Universidad de Los Andes, editado por Autores Varios. Bogotá: Departamento de Ciencia Política - Editorial Guadalupe Ltda.

Comisión de Estudios sobre la Violencia. 1987. Colombia: Violencia y Democracia. Bogotá: Universidad Nacional de Colombia. Comité editorial. 1987. "Presentación". Análisis Político 1 (mayo-agosto): 2-3.

Espinal, Milena. 1998. Ensambles de memoria: comunidades estudiantiles en la Universidad de los Andes. Tesis de grado, Departamento de Ciencia Política, Facultad de Ciencias Sociales, Universidad de los Andes.

González, Fernán, Ingrid Bolívar y Teófilo Vásquez. 2003. Violencia Política en Colombia. De la nación fragmentada a la construcción del Estado. Bogotá: CINEP.

Leal Buitrago, Francisco. 1984. Estado y Política en Colombia. Bogotá: Siglo XXI Editores.

Leal Buitrago, Francisco. 1994. "La profesionalización de los estudios políticos en Colombia". En El Estudio de la Ciencia Política en Colombia, editado por Rubén Sánchez. Bogota: Departamento de Ciencia Política, Universidad de LoS Andes, 93-124.

Leal, Francisco, Dora Rothlisberger y Rubén Sánchez. 1983. "El departamento de ciencia política". En La Ciencia Política y la Universidad de Los Andes, editado por Autores Varios. Bogotá: Departamento de Ciencia Política - Editorial Guadalupe Ltda., 37-57.

Leal Buitrago, Francisco y Germán Rey (eds.). 2000. Discurso y razón. Una historia de las ciencias sociales en Colombia. Bogotá: Tercer Mundo Editores - Ediciones Uniandes - Facultad de Ciencias Sociales - Fundación Social.

Murillo Castaño, Gabriel y Elisabeth Ungar Bleier. 2000. "Evolución y desarrollo de la ciencia política colombiana: un proceso en marcha". En Discurso y razón. Una historia de las ciencias sociales en Colombia, editado por Francisco Leal Buitrago y Germán Rey. Bogota: Tercer Mundo Editores - Ediciones Uniandes - Facultad de Ciencias Sociales Fundación Social, 213 - 223.

Oquist, Paul. 1980. Violence, Conflict and Politics in Colombia. New York: Academic Press.

Pécaut, Daniel. 1998. "La contribución del lepri a los estudios sobre la violencia en Colombia". Análisis Político 34 (mayoagosto): 71-89.

Postgrados. s. f. Departamento de Ciencia Política, Facultad de Ciencias Sociales, Universidad de Los Andes.

Sánchez, Rubén (ed.). 1994. El Estudio de la Ciencia Política en Colombia. Bogota: Departamento de Ciencia Política, Universidad de Los Andes.

Segura Escobar, Nora y Álvaro Camacho Guizado. 2000. "En los cuarenta años de la sociología colombiana". En Discurso y razón. Una historia de las ciencias sociales en Colombia, editado por Francisco Leal Buitrago y Germán Rey. Bogotá: Tercer Mundo Editores - Ediciones Uniandes - Facultad de Ciencias Sociales - Fundación Social, 179-197.

Serres, Francois. 2005. "Duelo de poder: Javerianos vs. Uniandinos dentro de la administración pública colombiana". El Tiempo 24 (febrero). 
Uricoechea, Fernando. 1999. La profesionalización académica en Colombia. Historia, estructura y procesos. Bogotá: Tercer Mundo Editores - IEPRI Universidad Nacional.

Ana María Bejarano es politóloga y profesora asistente del Departamento de Ciencia Política, Universidad de Toronto, Canadá. (E-mail: abejaran@utm.utoronto.ca)

María Emma Wills es politóloga y profesora asociada del Instituto de Estudios Políticos y Relaciones Internacionales de la Universidad Nacional de Colombia.

(E-mail: abril1959@yahoo.com ) 